\title{
Correlation of centrifugal pump vibration to unsteady flow under variable motor speed
}

\author{
J. Mele, A. Guzzomi and J. Pan
}

School of Mechanical and Chemical Engineering, The University of Western Australia, 35 Stirling Hwy, Crawley 6009, Australia

Received 7 January 2014, Accepted 15 May 2014

\begin{abstract}
Although condition monitoring of centrifugal pump bearings to infer faults is common practice, the relationship between a pump's vibration level and the unsteady flow within has not been extensively researched. The latter, however potentially provides the foundations for further developments in, pump design to increase performance, advanced predictive maintenance programs and, vibration monitoring techniques that can permit inference of pump efficiency states. This paper investigates the correlation between pump vibration and unsteady flow at different motor speeds. A test rig and a numerical CFD model were employed. It was found that flow-induced vibration in general increases with pump speed and was clearly linked to pump efficiency. It therefore seems possible to construct a model to deduce a pump's efficiency from its pressure and vibration levels, if the efficiency curve is known a priori. However, as the vibration levels are also dependent on the system's structural natural frequencies and modes, it seems that knowledge of these may also be needed in some instances. The work confirms that utilising a variable speed pump at lower pump speeds allows greater deviations from the design BEP without jeopardising the safety of the pump and should be considered for industrial use.
\end{abstract}

Key words: vibration based condition monitoring / unsteady flow / computational fluid dynamics / VFD/VSD / pump design

\section{Introduction}

Turbo machinery accounts for nearly $20 \%$ of the world's electrical energy, and up to $50 \%$ of energy in industrial plant operations [1]. The potential for cost savings provides technological companies with motivation to place considerable time and effort on pump design research.

Vibration is the leading cause of pump component failure and reduced pump performance. Pressure fluctuations interact with the volute casing and tongue region within the pump and give rise to unsteady forces, which, in turn, produce hydraulic vibration and noise. According to van Esch [2], pump design can only make further progress if effort is made to understand the internal flow. Further research is therefore required to better understand the relationship between vibration and unsteady flow inside centrifugal pumps for different operating conditions.

The most commonly used method to change pump speed is via a variable frequency drive (VFD) [3] or variable speed drive (VSD). VFD/VSD controlled pumps are becoming increasingly popular for industrial use. The

${ }^{a}$ Corresponding author: andrew.guzzomi@uwa.edu.au benefits include the possibility for significant energy savings, multi-purpose applications and improved reliability by means of speed reduction and avoiding part-flow operation [4]. The energy savings are achieved by operating the system at high efficiency levels by optimising the pump head, without the use of energy absorbing control valves. However, due to the absence of such demand in the past, literature is limited on VFD/VSD systems, especially in relation to the hydraulic vibration response. Kaiser et al. [4] point out that a potential downfall of variable speed pump systems is that operational frequencies may fall on natural frequencies of the pump and associated structure and cause excitations, which are typically avoided in single-speed pump systems.

The Spanish research group led by Gonzalez has performed leading research on centrifugal pumps. They note that flow inside a centrifugal pump is very complex and is dominated by geometrical constraints which give rise to highly three-dimensional phenomena [5]. Another source of unsteady flow arises from the suction surface of each of the blades, referred to as the jet-wake effect [6]. It gives rise to substantial pressure fluctuations, is a dynamic source of unsteady flow and is proportional to the flow rate. Brennen [6] also identifies the occurrence 
Table 1. Main characteristics of UWA test pump.

\begin{tabular}{lc}
\hline Impeller Diameter & $0.1915 \mathrm{~m}$ \\
Inlet Diameter & $0.050 \mathrm{~m}$ \\
Outlet Diameter & $0.025 \mathrm{~m}$ \\
Number of blades & 5 \\
\hline
\end{tabular}

of rotor-stator interactions that generate unsteady flow forces acting on the impeller producing radial forces on the impeller leading to vibration of the shaft.

The blade passing frequency (BPF) has been extensively studied and identified as a source of dynamic load and noise generation, through association with the fluiddynamic rotor-stator interaction phenomena. This frequency has typically been associated with the peak amplitude unsteady forces. In experiments performed by Marigorta [7] it was found that the predominant pressure peaks corresponded to the rotational frequency of the impeller and its harmonics, with significant response at the BPF. The results also indicated that the maximum pressure amplitudes corresponded to the low flow-rates. Yuan et al. [8] simulated and analysed a centrifugal pump using a CFD model and calculated the radial hydraulic forces. The vibration responses due to these forces were similar to those obtained by Marigorta [7] and Gonzalez \& Santolaria [9].

Pump efficiency is a universal measure of the pump performance and is defined as the ratio of the power supplied to the pump in relation to the power conveyed to the fluid. In most cases, centrifugal pumps are designed to work at the maximum efficiency level at a specific speed [6], known as the best efficiency point (BEP). Any deviation from the design point will result in energy wastage and manifest as excessive vibration and other mechanical instabilities. With the advent of VSD/VFD pump design may have to accommodate frequent operation at non constant speed.

This paper aims to identify, by means of experimentation and numerical methods, how centrifugal pump vibration and unsteady flow are related at different motor speeds.

\section{Experimental setup}

The test rig consists of a 4 -pole, $1.5 \mathrm{~kW}$ electric motor coupled to a Goulds 3196 MTX centrifugal pump. The motor's nominal speed is $1450 \mathrm{rpm}$. The characteristics of the test pump are identified in Table 1. Water is pumped to and from a reservoir as shown in the schematic (Fig. 1a).

The impeller has 5 front blades and 5 back blades (Fig. 1b); the back vanes serve to reduce the pressure on the shaft by producing a more balanced pressure distribution axially across the impeller. Prior to the commissioning of the pump, the impeller was machined down from an original diameter of $254 \mathrm{~mm}$ to $191.5 \mathrm{~mm}$. This was to reduce the head of the pump and match it more appropriately to the restrictive pump circuit. Typically
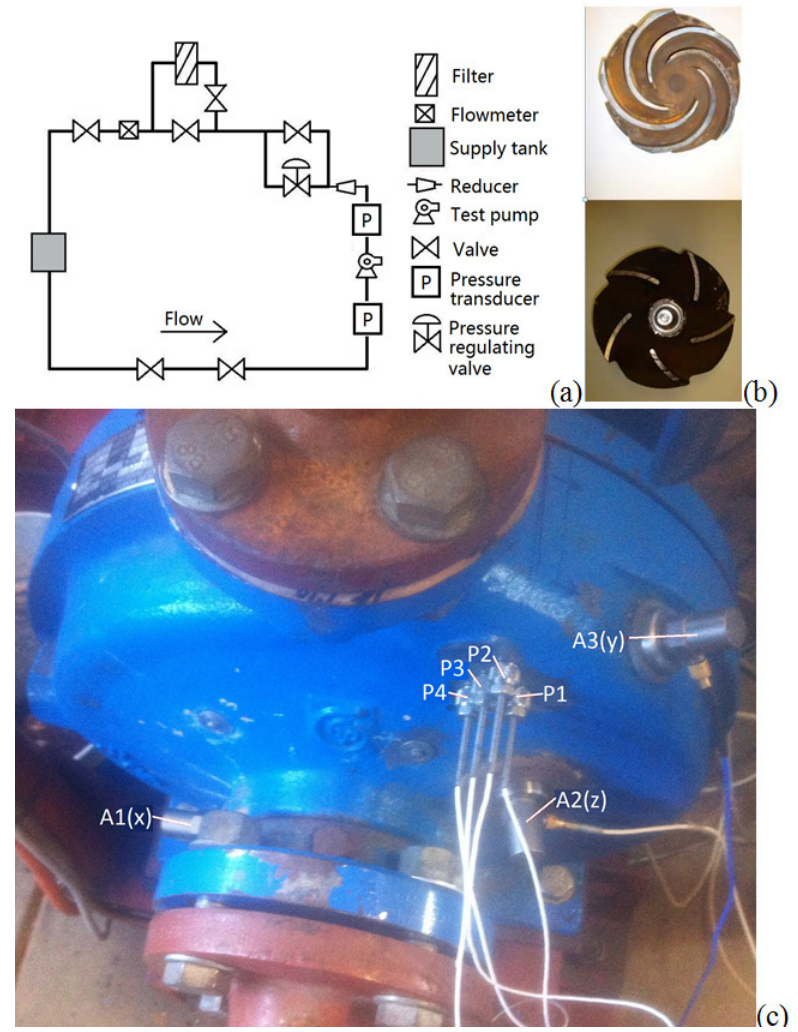

Fig. 1. UWA test: (a) rig schematic; (b) impeller-front, top and back, bottom; (c) accelerometers and pressure transducer locations on pump casing.

centrifugal pumps have a tongue gap to impeller radius ratio in the order of $0.05-0.10$ [10]; in this case, however, it is approximately 0.35 .

Three accelerometers were used for the experimental vibration measurement and were placed on the pump shroud, covering the 3 directions $(x, y$ and $z)$. The positioning of the accelerometers is shown in Figure 1c and the naming convention $\mathrm{A} 1, \mathrm{~A} 2$ and $\mathrm{A} 3$, corresponds to $x$, $z$ and $y$ respectively.

Based on previous studies and current literature [5,9], it is understood that the region surrounding the tongue of the centrifugal pump experiences the majority of unsteady forces. This is due to a stagnation experienced around the region as a result of flow being partitioned by the tongue. Hence three pressure transducers (P4, P3, $\mathrm{P} 2$ ) were placed in the volute, spanning radially across the tongue region. Another pressure transducer was placed in line with the tongue apex, but forward (P1). The positioning of the pressure transducers and naming convention are indicated in Figure 1c.

The motor speed was controlled by the VSD. Recent work by Guzzomi \& Pan [11] seems to be the first to highlight issues associated with the commonly accepted technique of using the motor speed to indicate the impeller speed. Their work investigated torsional vibration occurring across the flexible coupling. They concluded that ideally shaft angular velocity should be measured with a torsional laser vibrometer as close as possible to the impeller. 
Table 2. Inlet/outlet friction loss characteristics.

\begin{tabular}{lcc}
\hline & Outlet & Inlet \\
\hline Pipe length, $L[\mathrm{~m}]$ & 0.25 & 0.628 \\
Inner diameter, $D[\mathrm{~m}]$ & 0.021 & 0.046 \\
Relative roughness & 0.024 & 0.011 \\
Friction factor, $f_{D}$ & 0.06 & 0.05 \\
\hline
\end{tabular}

In the current study a hand held laser tachometer was used to determine the RPM of the exposed shaft close to the impeller. It was assumed that minimal loss in power occurs through the shaft coupling such that the average shaft speed corresponds to the impeller speed. Future tests will aim to incorporate torsional laser tachometry.

Tests were conducted at 10 different motor speeds between $0 \%$ to $100 \%$ of the safe engine speed (max $1600 \mathrm{rpm}$ ). The accelerometers used have a maximum frequency range of $10 \mathrm{kHz}$. This limited the sampling frequency to $20 \mathrm{kHz}$ due to Nyquist, which is noted to be more than adequate for the experiment results. The pressure and vibration signals were digitised and recorded to the PC system equipped with an 8-channel analogousdigital conversion card and the data recording software LabVIEW $^{\circledR}$. All the simulations were sampled at $20 \mathrm{kHz}$ for $10 \mathrm{~s}$. The FFT conversion was performed in MATLAB.

To measure the efficiency equation (1) was used in conjunction with data from the experiments.

$$
n=\frac{1000 g Q H}{I V \cdot n_{M} \cdot P F C}
$$

where the flow rate, $Q$, current, $I$, and voltage, $V$, were all measured directly. While the motor efficiency, $n_{M}$, and power factor correction, PFC, were taken as constant at 0.80 and 0.76 , respectively.

In equation (2), for the total head, $H$, the first two terms take into account the friction head according to the Darcy-Weisbach formula and the remaining terms relate, respectively, to the elevation difference, static head and dynamic head; the elevation difference was measured to be $0.4 \mathrm{~m}$.

$$
H=H_{D-W, \text { inlet }}+H_{D-W, \text { outlet }}+\Delta h+\frac{\Delta P}{\rho g}+\frac{\Delta v^{2}}{2 g}
$$

As noted in equation (2) the friction head was calculated for the inlet and outlet pipes between the inlet and outlet pressure gauges. Table 2 gives the relevant constants for the rig, with the friction factors found from the Moody diagram using the inner diameter and assuming a corroded steel pipe with a roughness of $0.5 \mathrm{~mm}$. Equation (3) was then evaluated using the parameters according to Table 2. The other terms in equation (2) were input from the measurements taken at each rotational speed.

$$
\left.H_{D-W}\right|_{\text {inlet/outlet }}=\left.f_{D} \frac{L}{D} \frac{v^{2}}{2 g}\right|_{\text {inlet/outlet }}
$$

\section{Numerical model setup}

The numerical model provides information on unsteady flow patterns, which are otherwise difficult to obtain from testing.

In order to generate the CFD model CAD models of the pump impeller and volute were required and thus detailed measurements of each component were taken. Six different parts: the base; back blades; volute; inlet; cavity case; and front blades were subsequently drawn.

ANSYS CFX ${ }^{\circledR}$ used the finite volume method and solved the Navier-Stokes (N-S) equations on vertex-based unstructured meshes. The N-S equations effectively relate the flow fields inside the centrifugal pump to the conservation laws resulting in the governing equations of fluid flow being the continuity, momentum and energy equations,

$$
\begin{aligned}
\frac{\partial \rho}{\partial t}+\nabla \cdot(\rho \mathbf{v}) & =0 \\
\frac{\partial \rho \mathbf{v}}{\partial t}+\nabla \cdot(\rho \mathbf{v} \otimes \mathbf{v}+p I-\tau) & =\rho \mathbf{f}_{e} \\
\frac{\partial \rho E}{\partial t}+\nabla \cdot(\rho \mathbf{v} E) & =\nabla \cdot(k \nabla T)+\nabla \cdot(\sigma \cdot \mathbf{v}) \\
& +\rho \mathbf{f}_{e} \cdot \mathbf{v}
\end{aligned}
$$

For low Mach number flow, the fluid is usually regarded as incompressible; the continuity equation and momentum equation can be solved independent of energy equation. The internal flow of the centrifugal pump was assumed to be incompressible owing to the fact that for the flow rates possible the Mach number would likely be $<0.3$. Due to the low pressure rise it was also regarded as isothermal. The model was split into three parts for the meshing, namely, the inlet, the impeller, and the volute. The meshing was done using the ICEM ${ }^{\circledR}$ CFD meshing software. For better accuracy and reduced runtime simulations, the structured hexahedra block mesh technique was preferred. This method was applied to the inlet and volute parts. Special attention was paid to the tongue region by increasing the element density and the boundary layers using inflation. The impeller geometry was seen to be too complex due to the existence of the front and back blades. An automated approach was therefore used to generate an unstructured mesh profile of the impeller. The fully assembled CFD model surface mesh is shown in Figure 2.

Direct numerical simulation (DNS) of the turbulent flow by solving the N-S equations is, in general, unrealistic for most engineering problems because of the very wide wavenumber-frequency spectra of turbulent flow. It was appropriate to solve the unsteady Reynolds averaged N-S equations (URANS) which are obtained by representing a flow property, e.g., velocity and pressure, in the $\mathrm{N}-\mathrm{S}$ equations as the sum of a steady mean component and a time-varying fluctuating component with zero mean value [12]. As a result, six additional unknowns, namely, the Reynolds stresses, are introduced in the time averaged momentum equations. Turbulence modelling procedures are of sufficient accuracy and generality to predict the Reynolds stresses. The standard $k$ - $\varepsilon$ model was adopted 


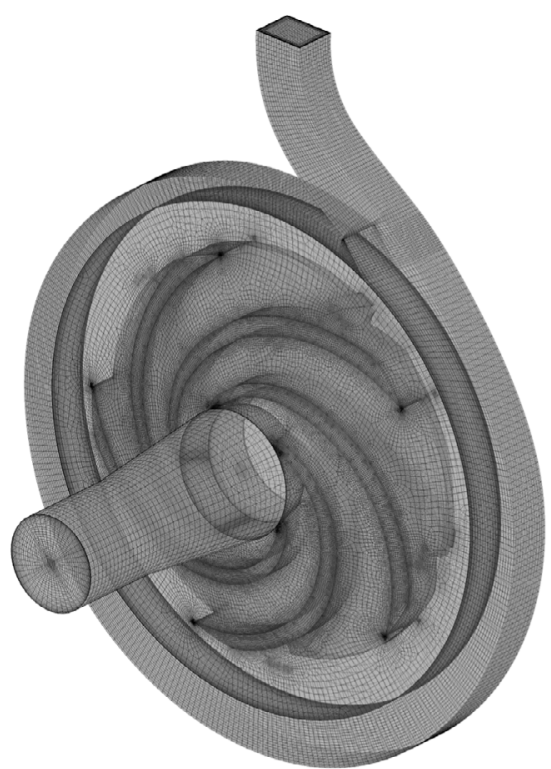

Fig. 2. Fully assembled pump surface mesh.

to depict the turbulent characteristics of the internal flow. Here $k$ and $\varepsilon$ refer to the turbulent kinetic and the rate of dissipation of turbulent kinetic energy per unit mass, respectively. The $k-\varepsilon$ model is well established and the most widely validated turbulence model [13].

The CFD model has a total of 1.86 million hexahedra elements, with 1.99 million nodes. Most CFD solvers demand the minimum face angle to be larger than 18 degrees and the maximum face angle to be smaller than 162 degrees; these criteria were comfortably satisfied.

The CFX setup had the impeller rotating at the desired motor speed, whilst the inlet and volute domains were set as stationary. As the impeller domain consisted of the impeller part as well as the cavity case part (which was physically stationary), the latter needed to be set to be counter-rotating to provide the correct fluid-boundary interactions. The boundary conditions were set using the inlet pressure and the outlet mass flow rate obtained during the experimental trials. The steady simulations used the frozen-rotor method [14]. A steady simulation was performed for each of the 10 experiment pump speeds and provided static pressure and velocity distributions as well as pump performance data. The results from the steady simulations served as the initial conditions for the unsteady calculations. The sliding-mesh technique was applied to the interfaces in order to simulate the unsteady interactions between the impeller and the volute. A complete impeller revolution was divided into 256 time steps and was related to the chosen motor speed to ensure accuracy and stability of the simulation. A total of 1024 time steps (4 full impeller revolutions) for each case was analysed. The unsteady simulation data provided information on the pressure fluctuations at the proposed monitoring points as well as dynamic pressure and velocity distributions for a full impeller rotation.

\section{Results and discussion}

\subsection{Experiment}

The total head and pump efficiency for each trial were calculated from the experimental data. Figures 3 and 4 show the filtered responses in which the peaks identified to vary with motor speed have been isolated. Figure 3 shows the acceleration responses of $\mathrm{A} 1, \mathrm{~A} 2$ and $\mathrm{A} 3$ respectively and the results show significant peaks at the rotational frequency $(\mathrm{RF}), \mathrm{BPF}$ and the $2 \mathrm{x} \mathrm{BPF}$. Other trends are evident as indicated on the graph. A1 and A3 ( $x$ and $y$ directions) show similar responses at the $\mathrm{RF}$ while A2 ( $z$ direction) is considerably different. The accelerometer sensor results show unusual responses varying with the motor speed; at approximately $12.8 \mathrm{x}$ RF and $16 \mathrm{x}$ RF. Such responses have not been identified in previous literature. It is possible these responses could be due to the modified impeller diameter. As such, investigations are currently underway with a full size original impeller. Figure 4 shows the FFT results from the pressure transducers (P1, P2, P3 and P4). During the experiments, P3 overloaded at motor speeds over $1300 \mathrm{rpm}$ as a result of the large pressure fluctuations at the tongue region due to the stagnation phenomenon.

The results show significant peak amplitudes at the $\mathrm{BPF}$ and $2 \mathrm{x}$ and $3 \mathrm{x}$. P3, which overloaded and is located closest to the tongue region, produced the largest response as expected due to the stagnation flow structure within the proximity. As identified from the acceleration FFT results, there are also interesting responses at $12.8 \mathrm{x} R$ and $16 \mathrm{x}$ RF. These frequencies are noticeably dependant on the motor speed. This indicates that the responses could be a result of unsteady flow.

Figure 3 shows an increase in RF vibration magnitude up to $1160 \mathrm{rpm}$ and then a decrease as the motor speed increases thereafter. The large response seems to indicate that it may be a natural frequency of the system. In an independent study by Johnstone [15], which experimentally investigated the receptances of the same test rig, a natural frequency of the rig was found near $20 \mathrm{~Hz}$. This agrees with studies performed by Marigorta [7].

It can be seen from both figures that the BPF response amplitudes generally increase with motor speed, even while approaching the BEP. The results seem to indicate the increasing BPF pressure magnitudes are due to the turbulent (proportional to fluid velocity) unsteady flow associated with the rotor-stator interactions as the fluid causes pressure fluctuations at the volute boundary opposing the blades. A1 and A2 both increase significantly with increasing motor speed. They are located in close proximity to the impeller. This would indicate the responses could be localised and are indicative of the jetwake induced hydraulic vibration, which would increase with impeller speed. However, A3, which is located closest to the outlet and tongue region, shows a steady decline in vibration response with increasing motor speed. This is seemingly more representative of the hydraulic vibration around the tongue region, which has been reported in previous literature to decrease with increasing pump 

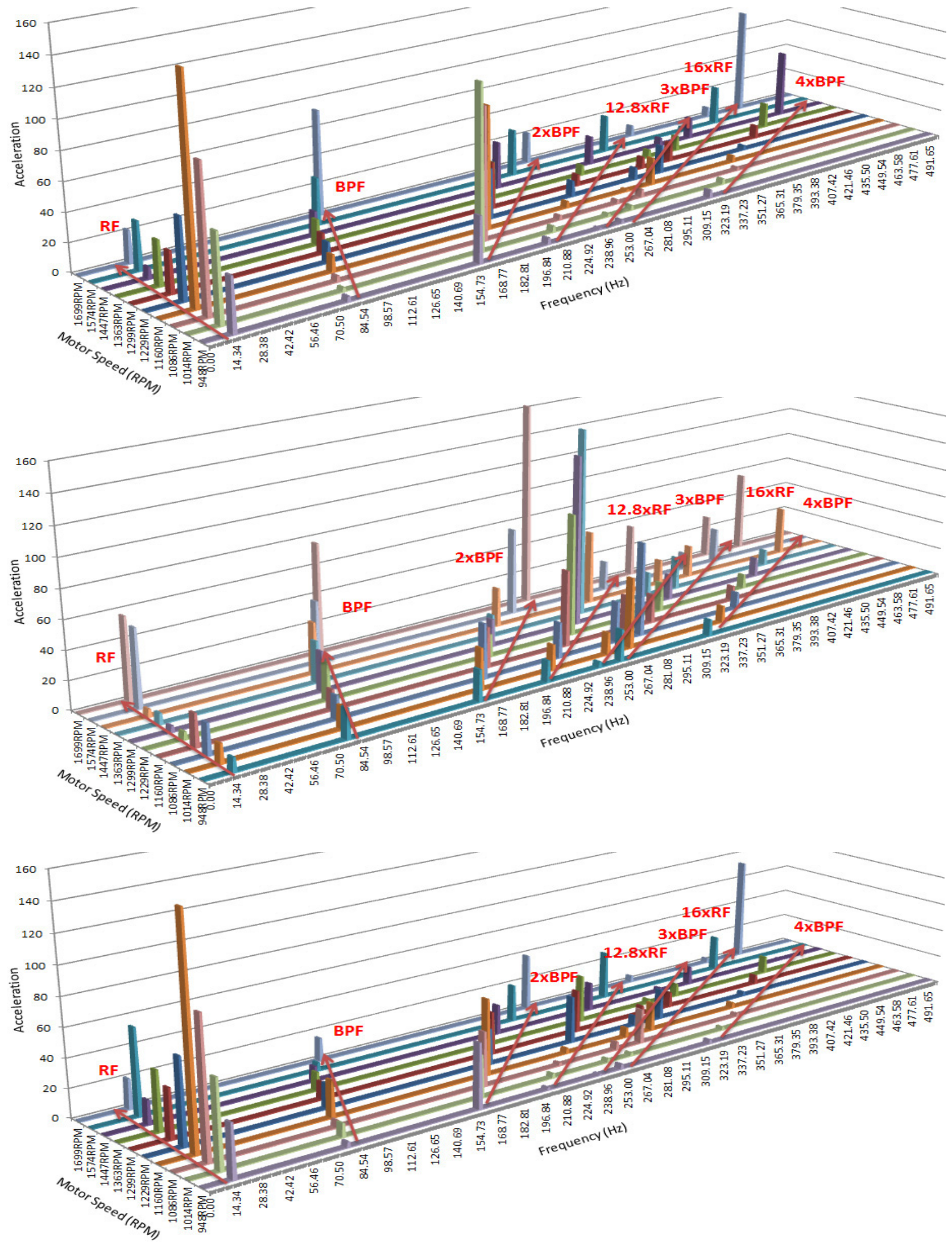

Fig. 3. Accelerometer non-dimensional 3D FFT response with varying motor speed: (a) A1; (b) A2, and; (c) A3. 

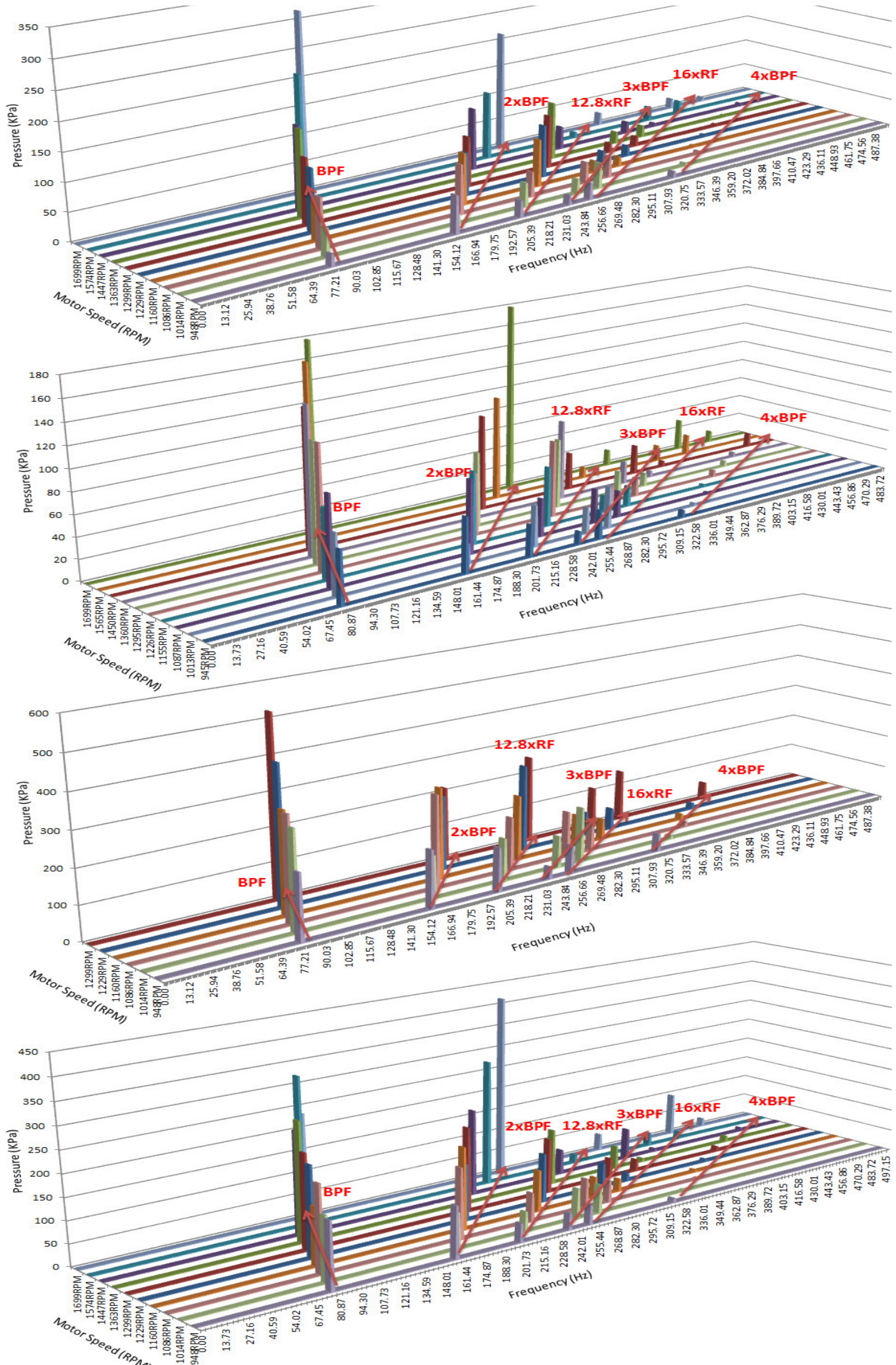

Fig. 4. Pressure Transducer FFT response with varying motor speed: (a) P1; (b) P2; (c) P3, and; (d) P4. 


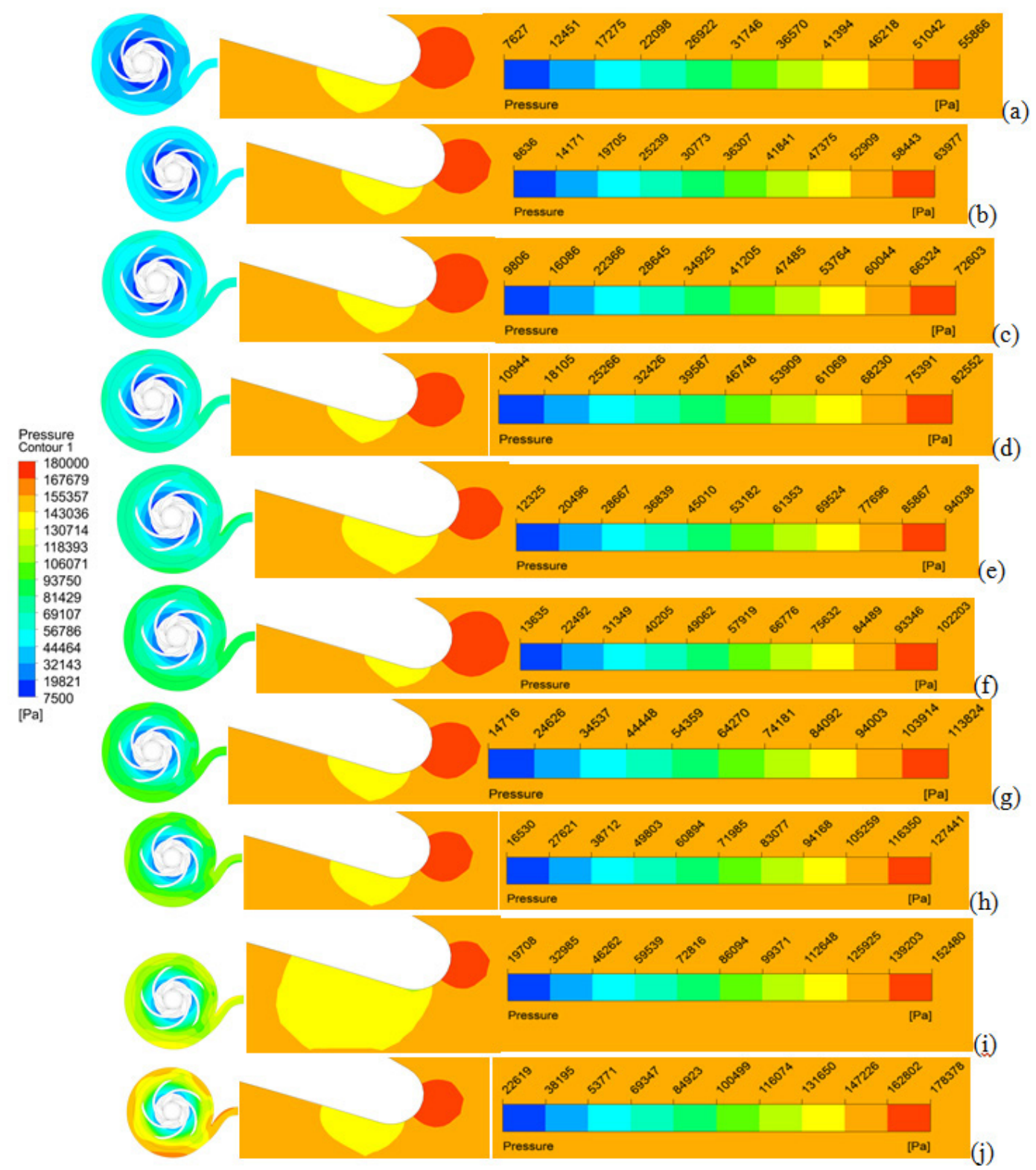

Fig. 5. Static pressure contours around the volute (left inlays) and at the tongue (middle inlays) at a motor speed [RPM] of: (a) 948; (b) 1014; (c) 1086; (d) 1160; (e) 1229; (f) 1299; (g) 1363; (h) 1447; (i) 1574; (j) 1699.

efficiency. The $2 \mathrm{x}$ BPF, provides a similar response to the BPF. Figure 4 shows the pressure response increasing with motor speed, while Figure 3 shows accelerometers A1 and A3 increasing initially, followed by an eventual decrease. This trend can be explained by similar reasoning to that used to explain the BPF. The response increases initially due to the increasing system energy, but as the pump approaches the BEP, the response decreases to a minimum. This again gives evidence that the BPF and the harmonics are related to the hydraulic vibration and that the response at the tongue decreases as the pump becomes more efficient. This reasoning has also been confirmed by Guzzomi and Pan [11] in regard to the BPF component of the torsional vibration signature around the BEP.

\subsection{CFD model}

The CFD model was used to help understanding of the pressure distribution in the pump, especially around the blades and tongue. Steady simulations using the frozenrotor method were performed for each motor RPM with the measured experimental inlet/outlet pressures as the boundary conditions. The pressure contour plots for each simulation were produced to analyse the change with motor speed and are shown in the left hand column inlays of Figure 5. It is evident from the results that the overall pressure magnitude increases with pump speed.

The steady simulations and static pressure profiles confirmed the highest pressure region inside the pump to be located around the tongue region, due to the 

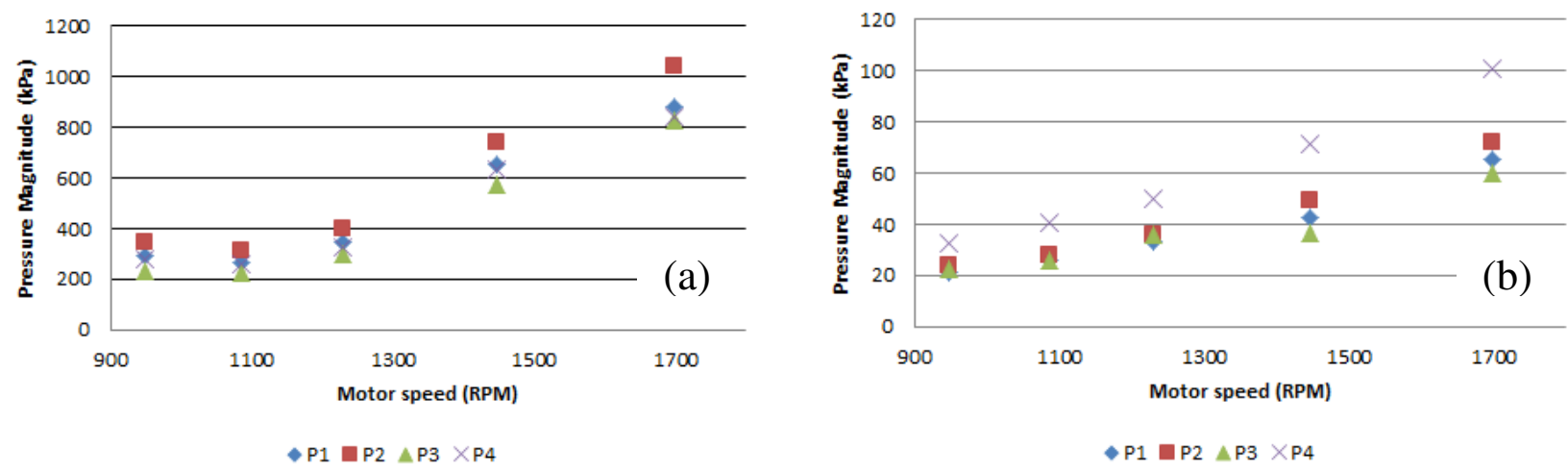

Fig. 6. Numerical pressure response for each of the monitoring points with varying RPM at: (a) BPF, (b) $2 x$ BPF.

stagnation of flow. This agreeds with experimental results and previous literature. Other evident high pressure regions are the zones on the volute walls opposing each of the impeller blades. This is evidence of the unsteady flow phenomenon mentioned earlier as the jet wake effect. It is due to fluid flow interactions between the fluid and the volute boundary walls and increases in magnitude as the motor speed increases.

The middle column inlays of Figure 5 show the pressure contours localised to the tongue region for the various speeds. The contour plot scale is specific to each motor speed to best illustrate the high and low pressure regions. Each speed case shows a high pressure zone around the apex of the tongue, where the stagnation region is expected, as well as a low pressure zone on the impeller side of the tongue, where fluid escapes after the collision and gets sucked back into the impeller. The size of the stagnation zone reduces with increasing motor speed, which is concurrently increasing the pump efficiency. This suggests that the system wastes less energy on hydraulic vibration at the tongue region and the flow is more freely able to pass through to the outlet. It can also be noticed that the high pressure becomes more central to the tongue apex with increasing motor speed, which agrees with the aforementioned conclusion that the complex flow structure at the tongue region impedes less flow as the pump system becomes more efficient.

The unsteady simulations yielded data from the pressure monitoring points and were able to provide information for the pressure fluctuation frequency responses to compare to the experimental results. The results of interest were the responses at the $\mathrm{BPF}$ and $2 \mathrm{x} \mathrm{BPF}$ and are displayed in Figure 6. A similar general trend with an increasing response as the motor speed is increased is observed. This trend agrees with the results from the pressure transducer. However, it is noticed that the monitoring point corresponding to $\mathrm{P} 3$ produces the smallest response, which is in conflict with the experiment results.

The efficiency from the experimental trials and the numerical simulations are compared in Figure 7. The curves increase at a similar rate with only minor fluctuations over the motor speeds. This indicates that the CFD model used is reliable and an accurate representation of the test

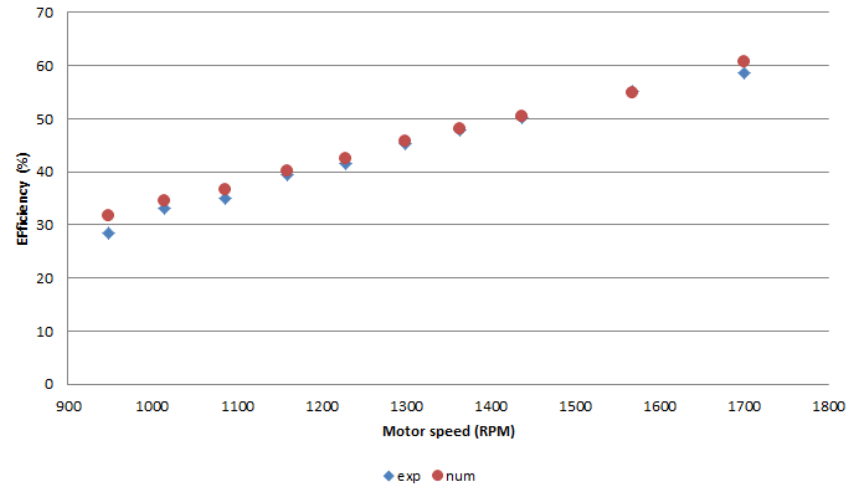

Fig. 7. Efficiency vs. RPM experiment and numerical model comparison.

pump internal flow. Both curves indicate that the pump increases in efficiency with increasing motor speed, and it is observed that the pump has not yet reached the BEP.

The responses from the pressure transducers and the numerical monitoring points are displayed in Figure 8, at the BPF, and Figure 9, at 2x the BPF. For both BPF results similar results for low frequencies are shown which drift further apart as the speed increases. On the other hand, at $2 \mathrm{x}$ the BPF the numerical method struggles to replicate the experiment data. This could due to a number of reasons. One being that the numerical model monitoring points did not exactly replicate the positions of the pressure transducers as the CFD model did not allow the monitoring points to be located flush so they were placed $0.1 \mathrm{~mm}$ off. Another possibility being that the complexity of the flow introduced by the presence of the back vanes could not be simulated by the CFD model. It is also probable that the solver was not able to capture the higher frequency phenomena as alluded to in Figure 8 and confirmed in Figure 9. Aside from adopting a more sophisticated solver, work is currently underway investigating the effect of the proximity of the constant boundary conditions. Despite these obvious shortcomings, it is interesting to remember how well the efficiency could be predicted (Fig. 7).

It is quite evident from the pressure transducer results that the motor speed increases the magnitude of 
P1

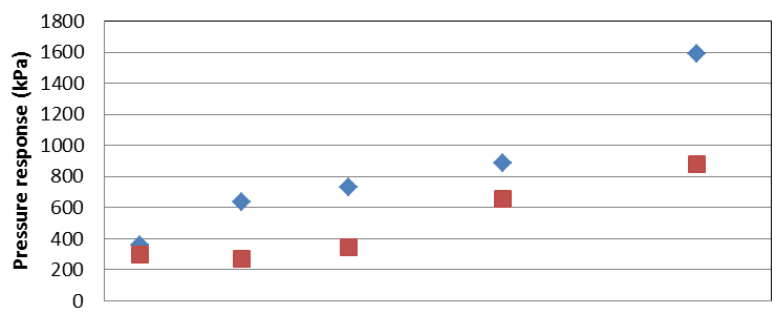

P4

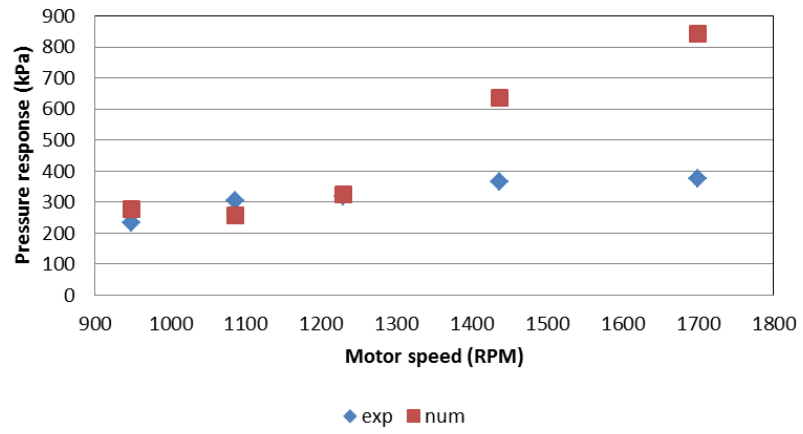

P2

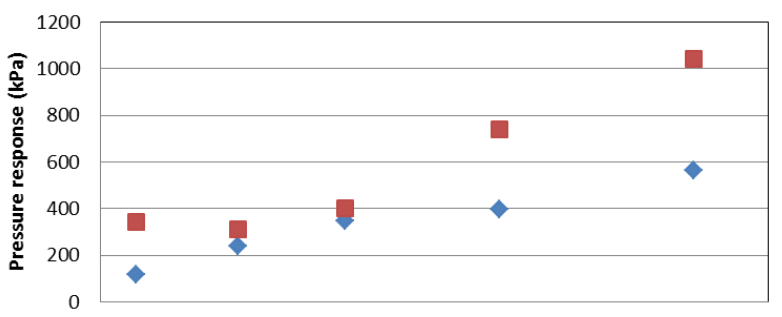

P3

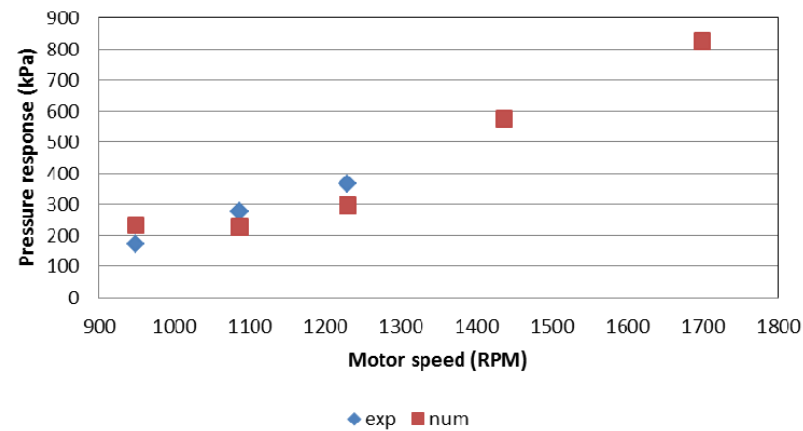

Fig. 8. Experimental and numerical pressure fluctuation magnitude at the BPF with varying rpm.

P1

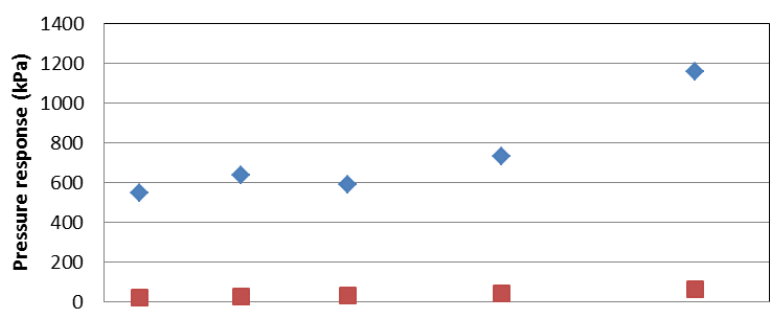

P3

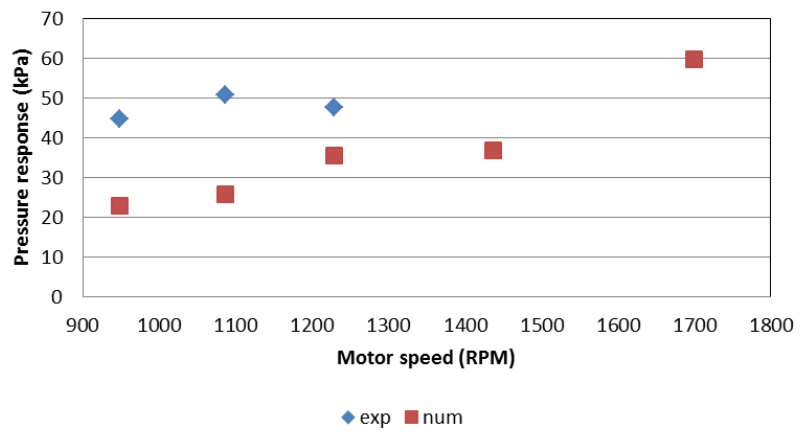

P2

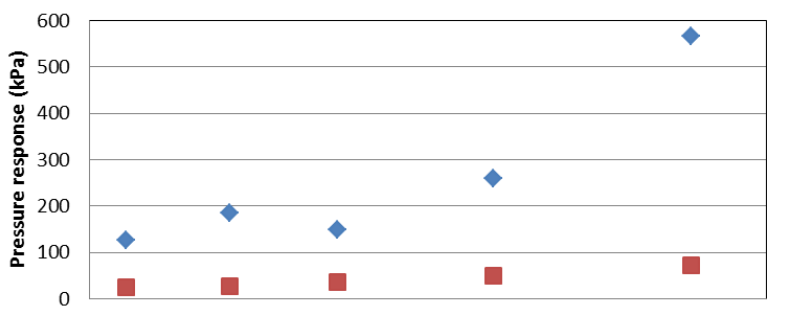

P4

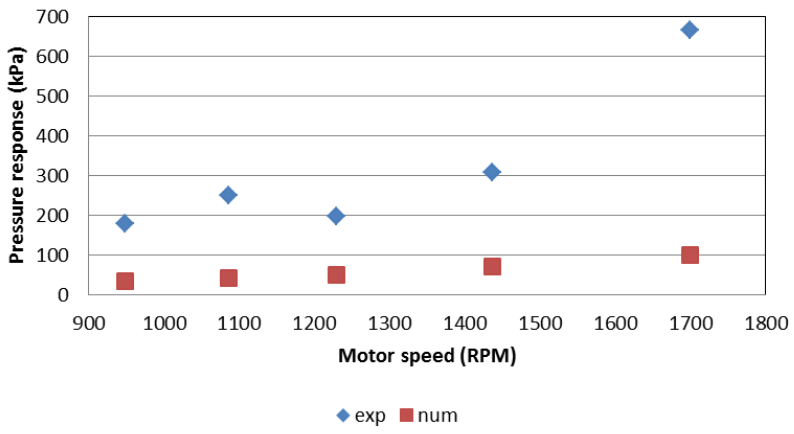

Fig. 9. Experimental and numerical pressure fluctuation magnitude at the $2 \mathrm{x}$ BPF with varying rpm.

the response significantly due to the addition of energy. However, from the CFD, as the BEP is approached, the stagnation region decreases in size and the origin moves more central to the tongue tip. This would comply with the vibration response results, which seem to decrease as the motor speed approaches the BEP, as the smaller stagnation zone would cause less disruption to the flow path. Moving away from the BEP, would appear to produce a general increase in hydraulic vibration response.

\section{Conclusions and future work}

It seems that the hydraulic induced vibration generally increases with increasing motor speed which makes physical sense as more energy is fed into the system, and hence a greater amount of pressure fluctuations can induce vibration responses. It seems the hydraulic vibration induced by the unsteady flow from the stagnation at the tongue region significantly affects the efficiency of the 
pump with the vibration reducing as the pump becomes more efficient. This was supported by the results from the numerical model. The numerical model proved to accurately represent the test pump and gave insight into the unsteady flow fields within the pump. The numerical results showed the existence of numerous unsteady flow fields, namely, the jet wake effect and the stagnation phenomena at the tongue region. The static pressure distributions showed that the stagnation region reduces in size as the pump becomes more efficient, suggesting the system wastes less energy. The FFT results at the BPF agree with the aforementioned conclusion, as the vibration response reduces with increasing motor speed.

It appears that a model could be constructed to deduce a pump's efficiency from its pressure and vibration levels, if the efficiency curve were known. The use of VFD/VSD at lower pump speeds appears to allow greater deviations from the design $\mathrm{BEP}$ without jeopardising the safety of the pump and is considered useful for industrial use.

Acknowledgements. The authors acknowledge the financial support from CIEAM 2. The assistance of Dr Cai with the CFD modelling is also gratefully acknowledged.

\section{References}

[1] US DOE, 2004, "Variable Speed Pumping - A guide to Successful Applications", US Department Of Energy

[2] B.P.M. Van Esch, "Simulation of three-dimensional unsteady flow in hydraulic pumps", Enschede, Netherlands, 1997

[3] UNEP, "Electrical Energy Equipment: Pumps and Pumping Systems", United Nations Environmental Programme, 2006
[4] T.F. Kaiser, R.H. Osman, R.O. Dickau, Analysis Guide for Variable Frequency Drive Operated Centrifugal Pumps, Proceedings of the Twenty-Fourth International Pump Users Symposium, 2008, pp. 81-106

[5] J. González-Pérez, J. Parrondo, C. Santolaria, E. Blanco J. Gonzales, Steady and Unsteady Radial Forces for a Centrifugal Pump With Impeller to Tongue Gap Variation, ASME J. Fluids Eng. 128 (2006) 454-462

[6] C.E. Brennen, "Hydrodynamics of Pumps", Cambridge University Press, 2011

[7] E.B. Marigorta, Fluid-Dynamic Radial Forces at the Blade-Passing Frequency in a Centrifugal Pump with Different Impeller Diameters, 2006, ASME J. Fluids Eng.

[8] J. Yuan, Y. Liang, S. Yuan, H. Xiong, J. Pei, Analysis of Flow-Induced Vibration of the Volute of a Centrifugal Pump Based on Finite Element Method, Jiangsu University China, 2010

[9] J. Gonzales, C. Santolaria, Unsteady Flow structure and Global Variables in a Centrifugal Pump, 2006, ASME J. Fluids Eng

[10] G. Rzentkowski, Generation and control of pressure pulsations emitted from centrifugal pumps: a review, ASMEPUBLICATIONS-PVP, 1996

[11] A.L. Guzzomi, J. Pan, Correlation of pump efficiency and shaft torsional vibration using torsional laser vibrometry, Acoustics 2012 Fremantle: Acoustics, Development and the Environment, Fremantle, Australia, 2012, pp. 1-6

[12] A.L. Guzzomi, J.-C. Cheng, D. Reeve, Rotating Machinery Health Manager (UWA Pump Stethoscope System), Project Report, Cooperative Research Centre for Infrastructure and Engineering Asset Management, 2013

[13] H.K. Versteeg, W. Malalasekera, An introduction to computational fluid dynamics The finite volute method, 2nd edition. Essex, UK, Pearson Education Limited, 2007

[14] ANSYS, CFX-Solver Theory Guide, 2011

[15] S. Johnstone, The vibration of a centrifugal pump, School of Mechanical and Chemical Engineering, The University of Western Australia, Honours thesis, 2012 